\title{
Posturographic examination of body balance in patients with Chiari type I malformation and correlation with the presence of syringomyelia and degree of cerebellar ectopia
}

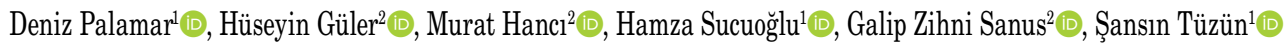 \\ ${ }^{1}$ Department of Physical Medicine and Rehabilitation, İstanbul University, Cerrahpaşa Medical Faculty, İstanbul, Turkey \\ ${ }^{2}$ Department of Neurosurgery, İstanbul University, Cerrahpaşa Medical Faculty, İstanbul, Turkey
}

Received: August 07, 2017 Accepted: November 10, 2017 Published online: March 11, 2018

\begin{abstract}
Objectives: We aimed to evaluate balance using the Tetrax Interactive Balance System (TIBS) in patients with Chiari type I malformation (CM-I) and to assess their fall risk with other clinical parameters.

Patients and methods: This cross-sectional, prospective study was conducted at physical medicine and rehabilitation outpatient clinics and neurosurgery department of a university hospital between December 2013 and December 2014. The study included a total of 36 patients ( 31 females, 5 males; mean age $40.6 \pm 10.0$ years; range, 18 to 60 years) with CM-I. Dynamic balance was assessed using the Berg Balance Scale (BBS), and posturographic examinations were performed using the TIBS.

Results: The mean Fall Risk Index (FI) value was $42.4 \pm 29.8$ and the mean BBS score was $55.5 \pm 1.5$. The most common complaints were neck pain $(94.4 \%)$, headache $(88.9 \%)$, paresthesia (86.1\%), fatigue $(83.3 \%)$, and vertigo $(80.6 \%)$. The mean tonsillar herniation below the foramen magnum was $8.7 \pm 3.8(\mathrm{~mm})$. Of the patients, $15(42.85 \%)$ had syringomyelia. The degree of tonsillar ectopia was statistically significantly lower in patients with syringomyelia $(\mathrm{p}<0.05)$. The BBS scores were near identical among the patients with or without syringomyelia, although the FI values were lower in the patients with syringomyelia. Comparing cerebellar ectopia $\leq 9 \mathrm{~mm}$ versus $>10 \mathrm{~mm}$, the FI values were slightly higher in the patients with ectopia $>10 \mathrm{~mm}$, although there was no statistically significant difference in the FI or BBS values.

Conclusion: Our study results suggest that CM-I can decrease the body balance stability and, thus, increase the fall risk. Evaluation of balance in patients with CM using TIBS static posturography is a simple and effective technique.
\end{abstract}

Keywords: Balance; chiari type I malformation; fall risk; posturography; Tetrax Interactive Balance System.

The term Chiari malformation (CM), also known as the Arnold-Chiari malformation, refers to caudal displacement of the cerebellar tonsils through the foramen magnum. Although four types of CM have been described to date, types I and II account for the majority of the clinical cases. ${ }^{[1]}$ The criterion for Chiari type I malformation (CM-I) is ectopia of the cerebellar tonsil $>5 \mathrm{~mm}$ below the foramen magnum. ${ }^{[1]}$ Syringomyelia of the cervical or cervicothoracic spinal cord may be seen and is typically present in 30 to $70 \%$ of patients with $\mathrm{CM} \cdot{ }^{[1-3]}$ There are several non-specific symptoms of CM-I including headache, neck pain, gait disturbances, muscle weakness, numbness and other abnormal feelings in the arm and legs, visual disturbances, tinnitus, dizziness, dysphagia, hearing loss, and vomiting. ${ }^{[4]}$

To maintain the body balance, integration of the visual, vestibular, and somatosensory systems is essential. ${ }^{[5]}$ Posturographic examinations with the Tetrax Interactive Balance System (TIBS; Tetrax Sunlight Medical Ltd., Ramat Gan, Israel) can be used to reliably assess the balance and stability. Balance disorders are commonly seen in the course of CM. Falls resulting in fracture or other musculoskeletal injuries have substantial personal and social burden worldwide along with immobilization and morbidity. ${ }^{[6,7]}$

Corresponding author: Deniz Palamar, MD. İstanbul Üniversitesi Cerrahpaşa Tıp Fakültesi Fiziksel Tıp ve Rehabilitsyon Anabilim Dalı, 34098 Fatih, İstanbul, Turkey. e-mail: denizpalamar@gmail.com 
The evaluation of postural balance is extremely important to prevent falls. In patients with $\mathrm{CM}$, due to the compression of the cerebellum at the posterior fossa and foramen magnum the symptoms related to cerebellar dysfunction such as dizziness, imbalance, and dysmetria can be observed. Thus, early detection of gait problems and assessment of fall risk are important in CM to avoid secondary complications due to falls through balance rehabilitation.

To the best of our knowledge, there is no previous report on changes in body balance in CM patients. Given the gait disturbances in this disorder, we aimed to evaluate the balance and fall risk with static posturography in patients with CM.

\section{PATIENTS AND METHODS}

This cross-sectional, prospective study was performed in patients with CM who were followed at the neurosurgery department of our university hospital. A written informed consent was obtained from each patient. The study protocol was approved by the İstanbul University, Cerrahpaşa Medical Faculty Ethics Committee. The study was conducted in accordance with the principles of the Declaration of Helsinki.

In total, 36 patients ( 31 females, 5 males; mean age $40.6 \pm 10.0$ years; range, 18 to 60 years) who were diagnosed with CM-I were included in this study between December 2013 and December 2014. Exclusion criteria were as follows: having a neurological disease disrupting balance, medical treatment disrupting balance, a systemic disease such as renal failure or decompensated heart failure disrupting functional status, and any mental dysfunction disrupting cooperation.
Demographic and clinical characteristics were collected on admission and included age, sex, Body Mass Index (BMI), and symptoms related to CM (neck pain, headache, back pain, diplopia, hyperacusis, dysphagia, vomiting, paresthesia, vertigo, lassitude, urinary incontinence, syncope, dysphonia, and dysarthria). On neurological examinations, muscle strength, superficial sensation, deep tendon and pathological reflexes, and the Romberg test results were assessed.

All balance measurements were performed in the physiotherapy laboratory under the supervision of a physiatrist. Dynamic balance was measured using the Berg Balance Scale (BBS). The inter-rater reliability coefficients for the BBS was 0.83 for elderly residents. ${ }^{[8]}$ Posturographic examinations were performed using the TIBS..$^{[9]}$

The TIBS evaluates the static postural balance by recording vertical pressure fluctuations from both toe tips and from both soles, during standing position on the device. The system digitally save the data from these different measurement platforms simultaneously and document them as visual and numerical values. It has a dedicated software system and a computer which can provide all relevant data. The computer integrates and processed the inputs from the surfaces, and analyzes the patient's balance. The patient's fall risk is calculated by measuring the oscillation angle between the center of pressure and the center of the gravity. All patients were instructed to stand on with their barefoot on the marked platforms and not to talk or move during the test.

Eight different positions were evaluated: (i) head straight, eyes open, support solid, (ii) head straight,

Table 1. Signs and symptoms of patients

\begin{tabular}{lccccc}
\hline & \multicolumn{2}{c}{ Absent } & & \multicolumn{2}{c}{ Present } \\
\cline { 2 - 3 } \cline { 6 - 7 } & $\mathrm{n}$ & $\%$ & & $\mathrm{n}$ & $\%$ \\
\hline Neck pain & 2 & 5.6 & & 34 & 94.4 \\
Headache & 4 & 11.1 & & 32 & 88.9 \\
Back pain & 10 & 27.8 & & 26 & 72.2 \\
Diplopia & 29 & 80.6 & & 7 & 19.4 \\
Hypoacusis & 21 & 58.3 & & 15 & 41.7 \\
Dysphagia & 12 & 33.3 & & 24 & 66.7 \\
Vomiting & 27 & 75 & & 9 & 25 \\
Paresthesia & 5 & 13.9 & & 31 & 86.1 \\
Vertigo & 7 & 19.4 & & 29 & 80.6 \\
Fatigue & 6 & 16.7 & & 30 & 83.3 \\
Urinary incontinence & 29 & 80.6 & & 7 & 19.4 \\
Syncope & 30 & 83.3 & & 6 & 16.7 \\
Dysphonia & 25 & 69.4 & & 11 & 30.6 \\
Dysarthria & 22 & 61.1 & & 14 & 38.9 \\
\hline
\end{tabular}


Table 2. Findings according to the presence of syringomyelia

\begin{tabular}{|c|c|c|c|c|c|c|c|}
\hline & \multicolumn{3}{|c|}{ Without syringomyelia $(\mathrm{n}=21)$} & \multicolumn{3}{|c|}{ With syringomyelia $(n=15)$} & \multirow[b]{2}{*}{$p$} \\
\hline & Mean \pm SD & Median & Min-Max & Mean \pm SD & Median & $\operatorname{Min-Max}$ & \\
\hline Age (year) & $43.0 \pm 9.6$ & & & $37.2 \pm 9.9$ & & & 0.090 \\
\hline Body Mass Index & $28.5 \pm 5.1$ & & & $30.6 \pm 7.3$ & & & 0.309 \\
\hline The amount of ectopia (mm) & $10.0 \pm 3.9$ & & & $7 \pm 3.0$ & & & $0.013^{*}$ \\
\hline Berg Balance Scale & $55.1 \pm 1.9$ & 56 & $48-56$ & $55.9 \pm 0.3$ & 56 & $55-56$ & 0.145 \\
\hline Fall Risk Index & $47.3 \pm 32.7$ & 38 & $2-100$ & $35.6 \pm 24.5$ & 30 & $0-96$ & 0.289 \\
\hline
\end{tabular}

SD: Standard deviation; Min: Minimum; Max: Maximum.

eyes closed, support solid, (iii) head straight, eyes open, support soft (iv), head straight, eyes closed, support soft, $(v)$ head turned to the right, eyes closed, support solid, (vi) head turned to the left, eyes closed, support solid, (vii) head up, eyes closed, support solid, and (viii) head down, eyes closed, support solid. Using the TIBS, we assessed vertical pressure fluctuations on four separate elastic pads for 32 s. The Fall Risk Index (FI) were measured using this system. The FI is specified as a numeric value between 0 and 100 . A value of $0-35$ indicates a low risk, 36-57 indicates a moderate risk, and 58-100 indicates a high risk of fall.

\section{Statistical analysis}

Statistical analysis was performed using the IBM SPSS version 22.0 software (IBM Corp., Armonk, NY, USA). All data were presented in mean \pm standard deviation (SD). The normality of the data was assessed using the Shapiro-Wilk test. The independent samples t-test was used to compare normally distributed variables, while non-parametric Mann-Whitney $\mathrm{U}$ test was used to compare non-normally distributed variables (between-group comparisons). A $p$ value of $<0.05$ was considered statistically significant.

\section{RESULTS}

Of the patients, the mean BMI was $29.3 \pm 6.1 \mathrm{~kg} / \mathrm{m}^{2}$. The mean FI value as assessed by the TIBS was $42.4 \pm 29.8$, indicating a moderate risk of fall, while the mean BBS score was $55.5 \pm 1.5$ indicating low fall risk.
The proportions of signs and symptoms in the patients diagnosed with CM-I are provided in Table 1. The most common complaints were neck pain (94.4\%), headache $(88.9 \%)$, paresthesia $(86.1 \%)$, fatigue $(83.3 \%)$, and vertigo $(80.6 \%)$.

The mean tonsillar herniation below the foramen magnum was $8.7 \pm 3.8 \mathrm{~mm}$. Of the patients, 15 (41.67\%) had syringomyelia. Findings according to the presence of syringomyelia are provided in Table 2 . The degree of tonsillar ectopia was statistically significantly lower in patients with syringomyelia than in those without $(\mathrm{p}=0.013)$. The BBS values were almost identical among the patients with or without syringomyelia, although the FI values were lower in the patients with syringomyelia. Comparing cerebellar ectopia $\leq 9 \mathrm{~mm}$ versus cerebellar ectopia $>10 \mathrm{~mm}$, the FI values were slightly higher in the patients with ectopia $>10 \mathrm{~mm}$, although there was no statistically significant difference in the FI or BBS values (Table 3).

\section{DISCUSSION}

Signs and symptoms in patients with CM-I occur due to direct compression of the posterior fossa structures and altered cerebrospinal fluid flow dynamics at the craniocervical junction. The severity of the clinical signs is proportional to the impact on the flow of cerebrospinal fluid at the craniocervical junction, ${ }^{[10]}$ and abnormal cerebrospinal fluid flow patterns are detected significantly more often in patients with symptomatic CM-I than in those with asymptomatic tonsillar ectopia. ${ }^{[1]}$

Table 3. Comparison of patients according to degree of cerebellar ectopia (Group 1: $\leq 9 \mathrm{~mm}$, Group 2: $\geq 10 \mathrm{~mm}$ )

\begin{tabular}{|c|c|c|c|c|c|c|c|}
\hline & \multicolumn{3}{|c|}{ Group $1(n=23)$} & \multicolumn{3}{|c|}{ Group $2(n=13)$} & \multirow[b]{2}{*}{$p$} \\
\hline & Mean \pm SD & Median & Min-Max & Mean \pm SD & Median & Min-Max & \\
\hline Age (year) & $39.6 \pm 9.5$ & & & $42.3 \pm 11.0$ & & & 0.439 \\
\hline Body Mass Index & $30.3 \pm 6.2$ & & & $27.7 \pm 5.7$ & & & 0.217 \\
\hline Berg Balance Scale & $55.8 \pm 0.7$ & 56 & $53-56$ & $54.9 \pm 2.3$ & 56 & $48-56$ & 0.080 \\
\hline Fall Risk Index & $41.6 \pm 28.8$ & 38 & $0-100$ & $44 \pm 32.6$ & 38 & $0-100$ & 0.217 \\
\hline
\end{tabular}

SD: Standard deviation; Min: Minimum; Max: Maximum. 
Signs and symptoms of CM vary widely. The most common symptoms are headache, neck pain, gait disturbance, muscle weakness, numbness and other abnormal feelings in the arms and legs, visual disturbances, tinnitus, dizziness, dysphagia, hearing loss, and vomiting. ${ }^{[4]}$ However, diagnosis of the disease without radiological evaluation is difficult, as there is no specific finding. ${ }^{[4,12,13]}$ Due to the compression of the cerebellum at the posterior fossa and foramen magnum, the symptoms are related to cerebellar dysfunction. Typical complaints are dizziness, imbalance, and dysmetria. The most common symptoms in this study group were neck pain $(94.4 \%)$, headache $(88.9 \%)$, paresthesia $(86.1 \%)$, fatigue $(83.3 \%)$, and vertigo $(80.6 \%)$. In a retrospective study, Wu et al. ${ }^{[14]}$ evaluated 49 pediatric patients under 14 years of age. The most common symptoms were headache, neck pain, and ataxia. Also, the magnitude of tonsillar ectopia was found to be correlated with neurological severity scores, but not with other clinical measures.

Syringomyelia is present in 30 to $70 \%$ of CM-I patients. ${ }^{[1-3]}$ The progression of syringomyelia associated with CM-I is due to the action of the cerebellar tonsils, which partially occlude the subarachnoid space at the foramen magnum and act as a piston on the partially enclosed spinal subarachnoid space. ${ }^{[15]}$ Syringomyelia can be also associated with specific clinical signs and symptoms. Gait disturbance is one of the signs seen in patients with CM-I with syringomyelia. In our study, 15 patients $(41.67 \%)$ had syringomyelia, consistent with the literature reports. ${ }^{[1-3]}$

Postural instability is a major problem in neurological diseases and it increases the frequency of falls and their consequences. ${ }^{[16]}$ In CM-I patients, decreased balance control may lead to fall injuries that can result in serious physical, psychological, and social impairment. Thus, improving balance may reduce CM-I-related cerebellar symptoms and related fall injuries. On this basis, we assessed balance with static posturography in CM-I patients. To the best of our knowledge, this is the first reported study to assess balance practically in CM patients.

Posturography is a balance measurement tool that is used to quantitatively assess postural stability and postural responses. ${ }^{[9]}$ It is based on computerized elaboration of electronic signals emitted by four surfaces from both toe tips and both soles. In this way, investigation of the visual, somatosensory, motor and central nervous systems can be performed. ${ }^{[9]}$
Although previous studies regarding balance disorders used many different balance evaluation tests, the use of the TIBS to evaluate the balance functions has gained popularity in recent years. ${ }^{[17-20]} \mathrm{A}$ recent study has demonstrated the high test-retest reliability of TIBS in young healthy adults with low physical activity level. ${ }^{[21]}$

A study which investigates postural performance in cervical radiculopathy found statistically higher fall risks in patients with cervical root compression group, compared to the patients without cervical root compression. ${ }^{[22]}$ They interpreted these results as inadequate transmission of proprioceptive/ sensory information to the medulla spinalis. Also, posturographic assessment of body balance was used in neurological disorders and found to be a useful tool for the evaluation of balance. ${ }^{[18-20]}$ Other than neurological problems, visual impairments can also affect postural stability and increase FI during posturographic examination. ${ }^{[23]}$

A study investigating the effect of hemodialysis on balance found the FI score to be significantly higher after hemodialysis and they emphasized the risk of falling in all age groups of patients with renal insufficiency following hemodialysis. ${ }^{[24]}$ On the other hand, Akdeniz et al. ${ }^{[25]}$ highlighted the importance of hypovitaminosis of vitamin D for risk of falling. They found better balance control and decrease FI in patients with serum 25-OH-vitamin D levels higher than $50.0 \mathrm{nmol} / \mathrm{L}$ in women aged 60 and above. Besides the balance evaluation, the TIBS can also be used effectively for exercise applications in patients with risk of falling. ${ }^{[26,27]}$

Further evaluation of balance and treatment protocols in CM-I are required. In the present study, balance measurements were performed using the TIBS static posturography, which can also measure FI. The mean fall risk of the patients was $42.4 \pm 29.8$, indicating a moderate risk of fall. The FI values were lower in patients with syringomyelia; however, no statistically significant difference was seen between patients with or without syringomyelia $(p=0.289)$. Also, the BBS values were almost identical among the patients with or without syringomyelia $(\mathrm{p}=0.145)$.

The clinical presentation of CM-I is not always correlated with the degree of cerebellar tonsillar ectopia. ${ }^{[4,28]}$ In this study group, the degree of tonsillar ectopia was statistically significantly lower in patients with syringomyelia than in those without $(\mathrm{p}=0.013)$. However, no correlation was observed between the degree of cerebellar ectopia and clinical symptoms. 
Although there was no statistically significant difference in the FI or BBS values between the groups (patients with cerebellar ectopia $\leq 9 \mathrm{~mm}$ versus $>10 \mathrm{~mm}$ ), the FI values were slightly higher in patients with ectopia $>10 \mathrm{~mm}(\mathrm{p}=0.217)$.

Previously, the TIBS has been used to predict the fall risk in patients with diabetic neuropathy, cervical spinal injuries, Parkinson's disease, and multiple sclerosis, as well as to assess balance ability in stroke patients..$^{[17-19]}$ However, it was not used before to evaluate balance control in patients with CM. Therefore, this is the first reported study to use TIBS, and other methods, to demonstrate the importance of balance training in this patient group.

Based on our findings, CM-I can decrease body balance stability and, thus, increase the risk of a fall. We found a moderate risk of fall with posturographic evaluation in our study group. Posturographic examination of body balance with the TIBS is simple and practical. However, the main limitations to this study include its small sample size and lack of a healthy control group. Therefore, further large-scale studies involving healthy controls should be performed to confirm our findings.

In conclusion, our study results show that CM-I can decrease balance stability, and evaluating the balance of patients with CM using TIBS static posturography is a simple and effective technique. Also, balance training should be considered for patients with an impaired balance. Further evaluations of balance and treatment protocols are needed to improve balance control in this patient group to reduce related falls and complications.

\section{Declaration of conflicting interests}

The authors declared no conflicts of interest with respect to the authorship and/or publication of this article.

\section{Funding}

The authors received no financial support for the research and/or authorship of this article.

\section{REFERENCES}

1. Guinto, G, Zamorano C, Domínguez F, Sandoval B, Villasana O, Ortiz A. Chiari I malformation: part I. Contemp Neurosurg 2004;26:1-7.

2. Elster AD, Chen MY. Chiari I malformations: clinical and radiologic reappraisal. Radiology 1992;183:347-53.

3. Arnautovic A, Splavski B, Boop FA, Arnautovic KI. Pediatric and adult Chiari malformation Type I surgical series 19652013: a review of demographics, operative treatment, and outcomes. J Neurosurg Pediatr 2015;15:161-77.
4. Aitken LA, Lindan CE, Sidney S, Gupta N, Barkovich AJ, Sorel M, et al. Chiari type I malformation in a pediatric population. Pediatr Neurol 2009;40:449-54.

5. Gribble PA, Hertel J. Effect of lower-extremity muscle fatigue on postural control. Arch Phys Med Rehabil 2004;85:589-92.

6. Tinetti ME. Clinical practice. Preventing falls in elderly persons. N Engl J Med 2003;348:42-9.

7. Stel VS, Smit JH, Pluijm SM, Lips P. Consequences of falling in older men and women and risk factors for health service use and functional decline. Age Ageing 2004;33:58-65.

8. Berg K, Wood-Dauphinee S, Williams JI. The Balance Scale: reliability assessment with elderly residents and patients with an acute stroke. Scand J Rehabil Med 1995;27:27-36.

9. Kohen-Raz R. Application of tetra-ataxiametric posturography in clinical and developmental diagnosis. Percept Mot Skills 1991;73:635-56.

10. Krueger KD, Haughton VM, Hetzel S. Peak CSF velocities in patients with symptomatic and asymptomatic Chiari I malformation. AJNR Am J Neuroradiol 2010;31:1837-41.

11. Hofkes SK, Iskandar BJ, Turski PA, Gentry LR, McCue JB, Haughton VM. Differentiation between symptomatic Chiari I malformation and asymptomatic tonsilar ectopia by using cerebrospinal fluid flow imaging: initial estimate of imaging accuracy. Radiology 2007;245:532-40.

12. Greenlee JD, Donovan KA, Hasan DM, Menezes AH. Chiari I malformation in the very young child: the spectrum of presentations and experience in 31 children under age 6 years. Pediatrics 2002;110:1212-9.

13. Tisell M, Wallskog J, Linde M. Long-term outcome after surgery for Chiari I malformation. Acta Neurol Scand 2009;120:295-9.

14. Wu YW, Chin CT, Chan KM, Barkovich AJ, Ferriero DM. Pediatric Chiari I malformations: do clinical and radiologic features correlate? Neurology 1999;53:1271-6.

15. Heiss JD, Patronas N, DeVroom HL, Shawker T, Ennis R, Kammerer W, et al. Elucidating the pathophysiology of syringomyelia. J Neurosurg 1999;91:553-62.

16. Fukunaga JY, Quitschal RM, Doná F, Ferraz HB, Ganança MM, Caovilla HH. Postural control in Parkinson's disease. Braz J Otorhinolaryngol 2014;80:508-14.

17. Dehner C, Heym B, Maier D, Sander S, Arand M, Elbel M, et al. Postural control deficit in acute QTF grade II whiplash injuries. Gait Posture 2008;28:113-9.

18. Oppenheim U, Kohen-Raz R, Alex D, Kohen-Raz A, Azarya M. Postural characteristics of diabetic neuropathy. Diabetes Care 1999;22:328-32.

19. Kong SW, Jeong YW, Kim JY. Correlation between balance and gait according to pelvic displacement in stroke patients. J Phys Ther Sci 2015;27:2171-4.

20. Alpini D, Berardino FD, Mattei V, Caputo D, Schalek P, Cesarani A. Characteristics of multiple sclerosis patient stance control disorders, measured by means of posturography and related to brainstem lesions. Audiol Res 2012;2:e9.

21. Akkaya N, Doğanlar N, Çelik E, Aysşe SE, Akkaya S, Güngör HR, et al. Test-retest reliability of tetrax ${ }^{\circledast}$ static posturography system in young adults with low physical activity level. Int J Sports Phys Ther 2015;10:893-900. 
22. Tan J, Ye X, Yuan W. Comparing loss of anterior column height and cervical Cobb angle with three different types of anterior cervical discectomy and fusion. Zhongguo Xiu Fu Chong Jian Wai Ke Za Zhi 2006;20:367-71. [Abstract]

23. Kim SY, Moon BY, Cho HG. Changes in falling risk depending on induced axis directions of astigmatism on static posture. J Phys Ther Sci 2015;27:1971-3.

24. Erken E, Ozelsancak R, Sahin S, Yilmaz EE, Torun D, Leblebici B, et al. The effect of hemodialysis on balance measurements and risk of fall. Int Urol Nephrol 2016;48:1705-11.

25. Akdeniz S, Hepguler S, Öztürk C, Atamaz FC. The relation between vitamin $\mathrm{D}$ and postural balance according to clinical tests and tetrax posturography. J Phys Ther Sci
2016;28:1272-7.

26. Alptekin K, Karan A, Diracoglu D, Yildiz A, Baskent A, Eskiyurt N. Investigating the effectiveness of postural muscle electrostimulation and static posturography feedback exercises in elders with balance disorder. J Back Musculoskelet Rehabil 2016;29:151-9.

27. Hung JW, Yu MY, Chang KC, Lee HC, Hsieh YW, Chen PC. Feasibility of Using Tetrax Biofeedback Video Games for Balance Training in Patients With Chronic Hemiplegic Stroke. PM R 2016;8:962-70.

28. Meadows J, Kraut M, Guarnieri M, Haroun RI, Carson BS. Asymptomatic Chiari Type I malformations identified on magnetic resonance imaging. J Neurosurg 2000;92:920-6. 